\title{
Sliding Mode Control of Steerable Needles
}

\author{
D. Caleb Rucker, Member, IEEE, Jadav Das, Hunter B. Gilbert, Student Member, IEEE, \\ Philip J. Swaney, Student Member, IEEE, Michael I. Miga, Member, IEEE, \\ Nilanjan Sarkar, Senior Member, IEEE, and Robert J. Webster, III, Member, IEEE
}

\begin{abstract}
Steerable needles can potentially increase the accuracy of needle-based diagnosis and therapy delivery, provided they can be adequately controlled based on medical image information. We propose a novel sliding mode control law that can be used to deliver the tip of a flexible asymmetric-tipped needle to a desired point, or to track a desired trajectory within tissue. The proposed control strategy requires no a priori knowledge of model parameters, has bounded input speeds, and requires little computational resources. We show that if the standard nonholonomic model for tip-steered needles holds, then the control law will converge to desired targets in a reachable workspace, within a tolerance that can be defined by the control parameters. Experimental results validate the control law for target points and trajectory following in phantom tissue and $e x$ vivo liver. Experiments with targets that move during insertion illustrate robustness to disturbances caused by tissue deformation.
\end{abstract}

Index Terms-Image-guided interventions, medical robotics, needle steering, nonholonomic systems, sliding mode control, surgical robotics.

\section{INTRODUCTION}

$\mathbf{M}$ ANY useful clinical diagnoses and therapies throughout the human body require needle insertion, including biopsy, drug injection, regional anesthesia, brachytherapy, and thermal ablation, among others. Needle insertion is particularly important in the liver, where it is often used to diagnose and even treat diseases. For example, needle-based radio frequency ablation is an effective therapy for patients with hepatocellular carcinomas and colorectal cancer, providing a viable alternative to open surgery [1]. The efficacy of nearly any needle insertion

Manuscript received November 2, 2012; revised March 21, 2013; accepted June 17, 2013. Date of publication July 15, 2013; date of current version September 30, 2013. This paper was recommended for publication by Associate Editor M. Vendittelli and Editor G. Oriolo upon evaluation of the reviewers' comments. This work was supported in part by the National Science Foundation under Award IIS-1054331 and in part by the National Institutes of Health with Award R21 0EB11628 from the National Institute of Biomedical Imaging and Bioengineering and Award R01 CA162477 from the National Cancer Institute. The content of this paper is solely the responsibility of the authors and does not necessarily represent the official views of the National Science Foundation or the National Institutes of Health.

D. C. Rucker and M. I. Miga are with the Department of Biomedical Engineering, Vanderbilt University, Nashville, TN 37235 USA (e-mail: daniel.c.rucker@vanderbilt.edu; michael.i.miga@vanderbilt.edu).

J. Das is with Rockwell Automation, Inc., Shirley, NY 11967 USA (e-mail: jadav.das@gmail.com).

H. B. Gilbert, P. J. Swaney, N. Sarkar, and R. J. Webster, III are with the Department of Mechanical Engineering, Vanderbilt University, Nashville, TN 37235 USA (e-mail: hunter.b.gilbert@gmail.com; philip.j.swaney@ vanderbilt. edu; nilanjan.sarkar@vanderbilt.edu; robert.webster@vanderbilt.edu).

Color versions of one or more of the figures in this paper are available online at http://ieeexplore.ieee.org.

Digital Object Identifier 10.1109/TRO.2013.2271098 procedure depends on the accuracy of final needle tip position. Needle targeting accuracy is currently limited by factors such as needle and tissue deformation during insertion, registration error, patient movement, and reliance on human hand-eye coordination to insert the needle. While image-guided robotic needle placement systems (see [2] for an overview) can improve accuracy, they cannot completely eliminate the aforementioned sources of error, because they align the needle before the procedure and cannot control the path of the needle (i.e., "steer" it) during insertion. Interventions in the liver and other organs often involve obstacles for a needle, including blood vessels and bile ducts, making the ability to steer during insertion particularly important.

To address this, several needle steering strategies have been developed, including use of a robot to apply lateral forces and torques to the base of a relatively stiff needle during insertion [3], [4]. A variation on this approach is to "steer the target" by using external actuators to deform the tissue, keeping the target lined up in front of a straight needle during insertion [5]. A second approach uses precurved concentric elastic tubes, which can be rotated and translated within one another to change the shape of the needle shaft [6], [7]. The third, and perhaps most widely investigated method, uses relatively flexible needles with asymmetric tips. As the needle is inserted, asymmetric tissue forces cause the tip to deflect and follow a curved path through the tissue, which the flexible shaft follows. Asymmetric tip designs can be passive (e.g., using a beveled, prebent (kinked), precurved, or flexure tip) [8], [9] or actuatable [10], [11]. For passive asymmetric tipped needles (which we will henceforth refer to as tip-steered needles), the direction of tip deflection can be controlled by axially rotating the needle's shaft at the base during insertion.

Each of the above needle steering techniques exhibits different strengths and challenges, and none has yet emerged as the optimal solution in all applications. Base steering of stiff needles works well near the tissue surface, but steerability is reduced with depth, because the needle shaft must deform an increasing amount of tissue. Concentric tube needle steering does not rely on the surrounding tissue medium to achieve a curved path and, because of this, can be used in both free space [6], [7] and tissue-embedded applications [12]. However, it requires a priori design of the component tubes [13], and so does not offer as much variability in the final trajectory within tissue. Tip steering has the advantage of typically traveling along a "follow the leader" trajectory, where the shaft follows the path of the tip, and steerability is largely unaffected by insertion depth. Challenges with tip steering include the fact that interaction forces depend on tissue and needle mechanical properties [14], and nonholonomic constraints complicate the control problem. In this paper, 
we address these challenges by presenting a novel control law for tip-steered needles in 3-D.

\section{A. Prior Planning and Control Results for Tip Steering}

Kallem and Cowan [15] presented a controller that stabilizes a tip-steered needle to a desired subspace (e.g., a sphere or a plane in $\mathbb{R}^{3}$ ), without specifying where the needle goes within that subspace. To direct the needle in a plane, Reed et al. [16] coupled this controller to the path planners of Alterovitz et al. [17], to create an image-guided tip-steered planning/control system, which was experimentally validated. In this system, the planner decides when to activate a series of $180^{\circ}$ rotations of the base (thus aiming the tip in opposite directions within the plane to which it is stabilized). Recent work by Abayazid et al. [18] investigated the open-loop accuracy of planar needle deflection models and integrated image feedback in a control strategy, which was similarly based on a series of $180^{\circ}$ rotations. The effective curvature of the needle's path can be controlled by duty cycling the rotation of the base, which has been demonstrated in cadaver brain [19].

Generalized 3-D planners that account for tissue deformation and inhomogeneity have also been developed for tip-steered needles. These include planners based on diffusion [20], helical paths [21], and inverse kinematics [22]. The helical path framework of Hauser et al. [21] could be considered a planningas-control approach that continually computes a large number of potential needle paths during insertion and then selects one that minimizes the distance between the needle tip and desired target location. Each of these 3-D planners has been validated in simulation, but none have yet been demonstrated experimentally with a physical needle. Furthermore, any planner that cannot run in real time will require a trajectory-following 3-D controller such as the one we propose in this paper.

\section{B. Contributions}

Our primary contribution in this paper is a novel approach for the control of tip-steered needles in 3-D, which can be used to target a specific point or to follow a desired trajectory. Based on the well-known nonholonomic unicycle model for tip-steered needles, we formulate a sliding mode control law, which is computationally efficient and independent of any model parameters. Using Lyapunov analysis and piecewise solutions to the model differential equations, we prove that this causes the needle model to reach a desired target within a specified error bound expressed as a function of the control input speeds. This is the first control law for 3-D tip-steered needles of which we are aware whose convergence has been proven in this sense. We experimentally validate the proposed approach in phantom and ex vivo liver tissue for 3-D target points and trajectories, in the presence of tissue deformation, which causes unmodeled needle motion and target motion.

\section{REVIEW OF KINEMATIC MODEL}

We adopt the nonholonomic generalized unicycle model presented in [23] that describes the trajectory of a flexible asymmetric-tipped needle inserted into tissue. The model de- scribes the time derivative of a homogeneous transformation matrix $g(t)=\left[\begin{array}{cc}R(t) & \boldsymbol{p}(t) \\ 0 & 1\end{array}\right] \in \mathrm{SE}(3)$ containing the position $p \in \mathbb{R}^{3}$ and orientation $R \in \mathrm{SO}(3)$ (a $3 \times 3$ rotation matrix) of the needle tip as a function of two control inputs, i.e., $u_{1}$ (insertion velocity) and $u_{2}$ (axial rotation velocity), as follows:

$$
\begin{aligned}
& \dot{\boldsymbol{p}}=R\left[\begin{array}{c}
0 \\
0 \\
u_{1}
\end{array}\right] \\
& \dot{R}=R\left[\begin{array}{ccc}
0 & -u_{2} & 0 \\
u_{2} & 0 & -k u_{1} \\
0 & k u_{1} & 0
\end{array}\right]
\end{aligned}
$$

where $k$ is the curvature of the needle path (a positive constant model parameter). Given the input variables as a function of time, computation of the model-predicted needle path would entail integration of (1) as an initial value problem from the initial state $g_{0}$ forward in time to obtain $g(t)$.

\section{CONTROL APPROACH}

\section{A. Problem Statement}

Our objective is to establish a control law to select the input velocities $u_{1}$ and $u_{2}$ to drive the needle tip position $\boldsymbol{p}(t)$ to a desired point $\boldsymbol{p}_{d} \in \mathbb{R}^{3}$, given knowledge of the current needle tip frame $g$. Note that a desired orientation $R$ is not specified, and the elements of $R$ are by nature bounded, because (1) forces $R$ to evolve on $\mathrm{SO}(3)$.

\section{B. Error Definition and Error Dynamics}

For the purposes of formulating our control law and analyzing its convergence, we define a vector $\boldsymbol{e}(t)$ as

$$
\boldsymbol{e}(t)=\left[\begin{array}{lll}
e_{x} & e_{y} & e_{z}
\end{array}\right]^{T}=R^{T}(t)\left(p_{d}-p(t)\right)
$$

This is the positional error expressed in coordinates of the current needle tip frame at time $t$, and $e=0$ represents the desired system state. We can explicitly write the dynamics for $e$, which follows directly from the original model (1) as

$$
\dot{\boldsymbol{e}}=\left[\begin{array}{ccc}
0 & u_{2} & 0 \\
-u_{2} & 0 & k u_{1} \\
0 & -k u_{1} & 0
\end{array}\right] \boldsymbol{e}-\left[\begin{array}{c}
0 \\
0 \\
u_{1}
\end{array}\right]
$$

\section{Manifold Definition}

We define a manifold in the state space of (3) as $\sigma=0$, where

$$
\sigma=\operatorname{atan} 2\left(e_{x},-e_{y}\right)
$$

and atan 2 is the two argument arctangent function using the numerator first convention. Thus, $\sigma$ has a range of $[-\pi \pi]$ and is only zero when $e_{x}=0$ and $e_{y}<0$ simultaneously (i.e., when the target lies in the half plane defined by the $z$ - and negative $y$-axes of the needle tip frame, as depicted in Fig. 1). 

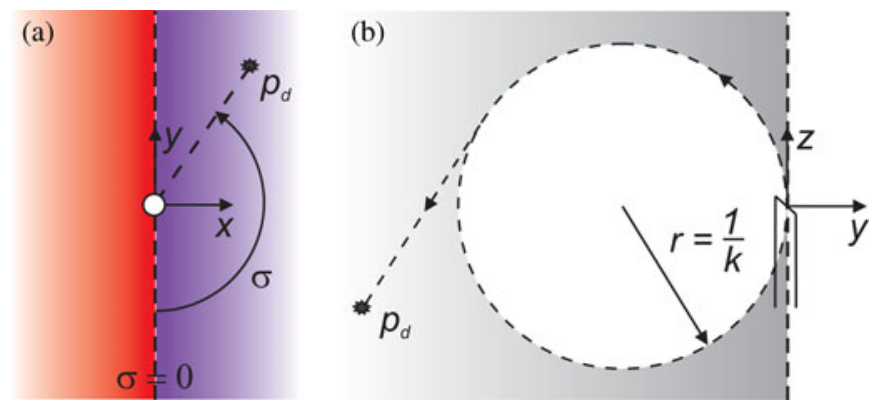

Fig. 1. (a) An arbitrary target position is projected onto the needle tip $x y$ plane. The angle $\sigma \in[-\pi \pi]$ is our metric for the (signed) distance away from the manifold. It is the minimum angle through which the needle tip would need to rotate in order to instantaneously satisfy $e_{x}=0$ and $e_{y}<0$. If the target projection resides in the blue region, then $\sigma>0$, while $\sigma<0$ in the red. (b) When $\sigma=0$, the target lies in the negative $y z$ plane of the needle tip frame. Under ideal controller action with $\lambda_{2} / \lambda_{1} \rightarrow \infty$, the system will stay on the manifold, and the trajectory of the needle will follow the arrows and converge to the target unless it is inside the dotted circle of radius $1 / k$, in which case the needle will circle the target until insertion is terminated.

\section{Control Law}

We now propose a control structure as

$$
\begin{aligned}
& u_{1}=\lambda_{1} \\
& u_{2}=\lambda_{2} \operatorname{sign}(\sigma)
\end{aligned}
$$

where $\lambda_{1}$ and $\lambda_{2}$ are positive constants, and the sign function simply returns -1 if the argument is negative, 1 if it is positive, and 0 if it is zero. This control approach is geometrically intuitive-we always want to rotate the needle toward a state in which it would curve toward the target when inserted, i.e., the manifold $\sigma=0$. It also guarantees that any desired limits on rotation and insertion speed are not exceeded.

\section{BEHAVIOR AS $\lambda_{2} / \lambda_{1} \rightarrow \infty$}

If $\lambda_{1}>0$, a geometric argument can be made that in the limit as $\lambda_{2} / \lambda_{1}$ approaches infinity, the system will reach $e=0$ in finite time for all target points located outside a particular region. In order to provide an intuitive understanding of the control law, we outline that argument for this ideal case before formally analyzing the more practical case of finite $\lambda_{2} / \lambda_{1}$.

As $\lambda_{2} / \lambda_{1} \rightarrow \infty$, rotations of the needle frame about its $z$-axis (due to the control input $u_{2}$ ) can be considered to happen instantaneously with respect to changes in the tip position. Therefore, the condition of $\sigma=0$ can be considered to be true at all times, since for any tip frame location and orientation, instantaneous rotation about the $z$-axis by an angle of $\sigma$ will bring the system to the manifold $\sigma=0$.

Now, let the following condition be satisfied at $t=0$ :

$$
\left(\sqrt{e_{y}^{2}+e_{x}^{2}}-\frac{1}{k}\right)^{2}+e_{z}^{2} \geq \frac{1}{k^{2}} .
$$

This condition constitutes the target location existing on or outside of the surface of a Horn's torus of radius $r=1 / k$ centered at the initial tip location and aligned with the initial $z$-axis. In the next instant, according to the control law, the action of $u_{2}$ will rotate the needle shaft by the minimum angle necessary to reach the sliding manifold, as shown in Fig. 1(a). Since the initial target position was outside the torus given by (6), the needle state now corresponds to that shown in Fig. 1(b). The control law then maintains the sliding condition continuously during insertion, and the needle traverses an arc of radius $r=1 / k$ until the tip tangent vector points directly through the target (i.e., $e_{x}=e_{y}=0$ and $e_{z} \geq 0$ ). This is a singular point in the definition of the manifold variable $\sigma$, and further insertion with no rotation would cause a finite change in $\sigma$. However, in the limit as $\lambda_{2} / \lambda_{1} \rightarrow \infty$, the control action will instantly correct this deviation and maintain the tangent state exactly. Thus, the needle tip must eventually pass through the target, reaching it in finite time due to the constant insertion velocity $\lambda_{1}$. Appropriate terminal behavior can be achieved by simply stopping the insertion when some criteria are met (e.g., when the needle tip is sufficiently close to the target, or when the error begins to increase as the needle passes the target).

If the target location was such that (6) was not satisfied at $t=0$, then the initial rotation to the sliding manifold would occur as before, but the tangent state will never be achieved due to the fixed curvature of the needle path. In this case, the needle tip would circle the target point on the sliding manifold until insertion is terminated, corresponding to a limit cycle. An informed choice of the needle's initial pose should always be made to ensure this situation does not occur.

\section{CONVERGEnCE FOR Finite $\lambda_{2} / \lambda_{1}$}

For finite $\lambda_{2} / \lambda_{1}$, the system can eventually reach a state where the error does not satisfy the torus condition, and some amount of tip error will be incurred even if the model is perfectly accurate. In this section, we analyze the behavior of the nonholonomic model system (1) under the control approach proposed in (5) for finite $\lambda_{2} / \lambda_{1}$. We show that the manifold $\sigma=0$ is always reached in finite time, and that it is a stable system state until the needle tip tangent points directly at the target. Then, we analyze the cycle of reaching the manifold state repeatedly until the target error reaches its minimum value, which we find an upper bound for as a function of the control input speeds.

\section{A. Model Solution for Constant Inputs}

If $u_{1}$ and $u_{2}$ are constant over an interval $t \in\left[\begin{array}{ll}0 & T\end{array}\right]$, then (1) and (3) are linear, and their solutions can be written as

$$
\begin{aligned}
\boldsymbol{p}(t) & =R(0) \boldsymbol{p}^{*}(t)+\boldsymbol{p}(0) \\
R(t) & =R(0) R^{*}(t) \\
\boldsymbol{e}(t) & =R^{* T}(t)\left(\boldsymbol{e}(0)-\boldsymbol{p}^{*}(t)\right)
\end{aligned}
$$

where $\boldsymbol{p}^{*}(t)$ and $R^{*}(t)$ are the solutions to (1) with $R(0)=I$ and $\boldsymbol{p}(0)=\mathbf{0}$, given by

$$
\boldsymbol{p}^{*}(t)=\frac{1}{q^{3}}\left[\begin{array}{c}
-k u_{1}^{2} u_{2}(S-q t) \\
k u_{1}^{2} q V \\
k^{2} u_{1}^{3} S+u_{2}^{2} u_{1} q t
\end{array}\right]
$$




$$
R^{*}(t)=\frac{1}{q^{2}}\left[\begin{array}{ccc}
q^{2}+u_{2}^{2} V & -u_{2} q S & -k u_{1} u_{2} V \\
u_{2} q S & q^{2} C & -k u_{1} q S \\
-k u_{1} u_{2} V & k u_{1} q S & q^{2}+k^{2} u_{1}^{2} V
\end{array}\right]
$$

and where

$$
\begin{aligned}
q & =\sqrt{k^{2} u_{1}^{2}+u_{2}^{2}} \\
S & =\sin (q t) \\
C & =\cos (q t) \\
V & =C-1 .
\end{aligned}
$$

Furthermore, $e(t)$ can be written as follows:

$$
\begin{aligned}
\boldsymbol{e}(t) & =R^{* T}(t) \boldsymbol{e}(0)+\frac{1}{q^{3}}\left[\begin{array}{c}
k u_{1}^{2} u_{2}(S-q t) \\
k u_{1}^{2} q V \\
-k^{2} u_{1}^{3} S-u_{2}^{2} u_{1} q t
\end{array}\right] \\
& =\left[\begin{array}{l}
a_{x} \\
a_{y} \\
a_{z}
\end{array}\right] S+\left[\begin{array}{l}
b_{x} \\
b_{y} \\
b_{z}
\end{array}\right] C+\left[\begin{array}{c}
c_{x} \\
0 \\
c_{z}
\end{array}\right] t+\left[\begin{array}{l}
d_{x} \\
d_{y} \\
d_{z}
\end{array}\right]
\end{aligned}
$$

where the coefficients are listed explicitly in the Appendix.

\section{B. Reaching the Manifold}

We can show that $\sigma=0$ is always reached in finite time by examining the error solution in (10) (since under the control law, the inputs are constant until the manifold is reached). First, we note that $\dot{e}_{x}=e_{y} \lambda_{2} \operatorname{sign}(\sigma)$ from (3) and (5). Since $\sigma$ has the same sign as $e_{x}$ (everywhere except $\sigma=\pi$, where $e_{x}=0$ ), then $\operatorname{sign}\left(\dot{e}_{x}\right)=-\operatorname{sign}\left(e_{x}\right)$ only when $e_{y}<0$. Thus, $e_{x}$ can only reach zero if $e_{y}<0$, which implies that the zeros of $\sigma(t)$ must correspond to the zeros of $e_{x}(t)$ in (10) with $u_{2}=\lambda_{2} \operatorname{sign}(\sigma)$.

Examination of (10) shows that $e_{x}(t)$ oscillates between two linear functions $f_{1}(t)=c_{x} t+d_{x}-\sqrt{a_{x}^{2}+b_{x}^{2}}$ and $f_{2}(t)=$ $c_{x} t+d_{x}+\sqrt{a_{x}^{2}+b_{x}^{2}}$, and $e_{x}(t)$ periodically equals each function with a period of $2 \pi / q$. Therefore, $e_{x}(t)$ must equal zero (and hence $\sigma=0$ ) at some time $t \in\left[\begin{array}{ll}\tau & \tau+2 \pi / q\end{array}\right]$, where $\tau \geq 0$ is the soonest time when $f_{1}(t)$ and $f_{2}(t)$ have different signs ( $\tau$ is guaranteed to exist because $\sqrt{a_{x}^{2}+b_{x}^{2}}>0$ and $\operatorname{sign}\left(c_{x}\right)=-\operatorname{sign}\left(e_{x}(0)\right)$ under the control law). We, therefore, conclude that the control law will force the system from any state to the manifold $\sigma=0$ within a time less than $2 \pi / q$ from $\tau$.

\section{Remaining on the Manifold}

In order to show that the system stays on the manifold once it has been reached, we employ the candidate Lyapunov function $L=\frac{1}{2} \sigma^{2}$. The derivative of $L$ is straightforward to obtain from (4) and (3) as follows:

$$
\dot{L}=\sigma \dot{\sigma}=\sigma\left(-\lambda_{2} \operatorname{sign}(\sigma)+k \frac{e_{z} e_{x}}{e_{x}^{2}+e_{y}^{2}} \lambda_{1}\right) .
$$

If $e_{y} \neq 0$, then there exists some neighborhood around $\sigma=0$ within which $\dot{L}$ is always negative, regardless of how large $e_{z}$ may be, since $e_{x} \rightarrow 0$ as $\sigma \rightarrow 0$. Therefore, $\sigma=0$ is a stable equilibrium point as long as $e_{y} \neq 0$. If the tangent state ( $e_{y}=e_{x}=0, e_{z} \geq 0$ ) is eventually reached, then $\sigma$ becomes undefined, and the system will leave the manifold. We discuss when this happens and the implications in the following section.

\section{Reaching the Tangent State}

We note that the axial rotation of the needle base at $t=0$ is somewhat arbitrary and could be chosen such that $\sigma=0$ before insertion begins. If we assume this initial rotation has been performed, then the control law will keep the system on the manifold (with $e_{x}=0$ ) until $e_{y}$ also becomes zero as we showed in the previous section.

We can determine from (10) that while on the manifold, $e_{y}$ must eventually become zero with $e_{z} \geq 0$ if and only if $a_{y}^{2}+$ $b_{y}^{2}>d_{y}^{2}$, which is identical to the torus condition given by (6) at $t=0$. Thus, for all targets outside the initial torus, the tangent state (i.e., $e_{x}=e_{y}=0, e_{z} \geq 0$ ) will eventually be reached.

\section{E. Cycle of Two Phases}

After the tangent state is reached, further insertion of the needle will cause $\sigma$ to jump from 0 to either $\pi$ or $-\pi$ in the next instant of time. Thus, the system trajectory will consist of two alternating phases, which may repeat multiple times. There will be a $\sigma \neq 0$ phase where the system reaches the manifold in a finite time and the needle is executing a helical trajectory. There will also be a $\sigma=0$ phase where the system is on the manifold and the needle is executing a circular trajectory. If the tangent state is reached in either phase, then the helical phase will begin again. If the target location is ever inside the torus (6) when the circular phase begins, then the system will stay in the circular phase until insertion is terminated.

\section{F. Upper Bound on the Error}

Assuming the tangent state has initially been reached, we would like to know the minimum value of $\|\boldsymbol{e}(t)\|$ (using the 2-norm) over the insertion, and we would also like to establish a rule for when insertion should be terminated. More generally, we are interested in finding an upper bound on $\min (\|\boldsymbol{e}(t)\|)$ for a given $\lambda_{1}$ and $\lambda_{2}$. For this quantity to be useful and convenient for choosing control parameters, we would like it to: 1) be as small as possible, preferably close to the least upper bound; 2) be easily expressed in a closed algebraic form; and 3) approach zero as $\lambda_{2} / \lambda_{1}$ approaches infinity.

If we consider the candidate Lyapunov function $L=\boldsymbol{e}^{T} \boldsymbol{e}$, its derivative is $\dot{L}=-e_{z} u_{1}$. This reflects the intuition that the error will decrease with insertion as long as the target is "in front of" the needle. When an initial tangent state is reached, $e_{z}(t)>0$; therefore, $e_{z}(t)$ must reach zero at some subsequent time (if not, then the system converges to $e=0$ anyway), and we should then stop inserting because the error will have stopped decreasing. In order to derive our upper bound on the minimum error, we now consider the two possible cases for the time when the system reaches $\left.e_{z}(t)=0: 1\right)$ during a helical phase, or 2) during a circular phase.

1) Case 1: Assign $t=0$ to be the time when the helical phase began (when the most recent tangent state was reached). Then, the manifold will be reached at some time $t \in\left[\begin{array}{ll}0 & 2 \pi / q\end{array}\right]$, since $f_{1}(0)$ and $f_{2}(0)$ have different signs. Since in case $1, e_{z}(t)=0$ before the manifold is reached, then $\min (\|\boldsymbol{e}(t)\|) \leq \max \left(\sqrt{e_{x}^{2}(t)+e_{y}^{2}(t)}\right)$ on the interval. Analysis of the derivative of the right-hand side then shows that its 
maximum occurs when $e_{y}(t)=0$ (which, according to (10), necessarily happens sometime within the interval); therefore, $\min (\|\boldsymbol{e}(t)\|) \leq \max \left(\left|e_{x}(t)\right|\right)$. By inspection of the form of $e_{x}(t)$ in (10), we see that $\max \left(\left|e_{x}(t)\right|\right) \leq\left|a_{x}\right|+\left|b_{x}\right|+\left|d_{x}\right|$. Using the coefficient formulas listed in the Appendix, and noting that $e_{x}(0)=e_{y}(0)=0$, this implies the following dimensionless error bound:

$$
\min (k\|\boldsymbol{e}(t)\|) \leq \frac{\bar{\lambda}}{\bar{q}^{3}}+2 k e_{z}(0) \frac{\bar{\lambda}}{\bar{q}^{2}}
$$

where $\bar{\lambda}=\frac{\lambda_{2}}{k \lambda_{1}}$ and $\bar{q}=\frac{q}{k \lambda_{1}}=\sqrt{1+\bar{\lambda}^{2}}$ are dimensionless quantities, and the left-hand side is the dimensionless error. Noting that the right-hand side is a function of $e_{z}(0)$, we can find an absolute upper bound by noting that since $e_{z}(t)=0$ somewhere on the interval, then $c_{z} 2 \pi / q+d_{z}-\left|a_{z}\right|-\left|b_{z}\right| \leq 0$. Again using the coefficients in the Appendix, we get

$$
k e_{z}(0) \leq \frac{2 \pi \bar{\lambda}^{2}+1}{\bar{q}\left(\bar{\lambda}^{2}-1\right)}
$$

which holds as long as the denominator is positive, which is equivalent to $\lambda_{2} / \lambda_{1}>k$. Substituting this result into (12) yields our final error bound for case 1 :

$$
\min (k\|\boldsymbol{e}(t)\|) \leq \frac{\bar{\lambda}}{\bar{q}^{3}}+\frac{4 \pi \bar{\lambda}^{3}+2 \bar{\lambda}}{\bar{q}^{3}\left(\bar{\lambda}^{2}-1\right)}=\beta_{1} .
$$

Thus, the needle is guaranteed to reach a sphere of radius $\beta_{1} / k$ around the target in case 1 (with the proviso that $\lambda_{2} / \lambda_{1}>k$ ). We note that $\beta_{1}$ is conservative because of the bounding approach taken (it is not the least upper bound), but it is easily expressed, and it does approach zero in the limit as $\bar{\lambda}=\frac{\lambda_{2}}{k \lambda_{1}}$ approaches infinity.

2) Case 2: As in case 1, let $t=0$ be the time when the most recent helical phase began, and let $t=\tau_{c}$ be the time when the current circular phase began (when the manifold was reached with $\left.e_{z}\left(\tau_{c}\right) \geq 0\right)$. Since, in case $2, e_{z}(t)$ becomes zero before the next helical phase would begin, we know that $e_{y}(t)=0$ is never reached, and thus, $\boldsymbol{e}\left(\tau_{c}\right)$ does not satisfy the torus condition (6). This fact already gives us an absolute error bound of $k\|\boldsymbol{e}(t)\| \leq$ 1 for case 2 , since the trajectory of the needle lies on the surface of the torus.

The minimum value of $\|\boldsymbol{e}(t)\|$ during the circular phase will occur when $e_{z}(t)=0$, and will equal the shortest distance from $\boldsymbol{e}\left(\tau_{c}\right)$ to the surface of the torus. Thus, a dimensionless error bound for the circular phase is

$$
\min (k\|\boldsymbol{e}(t)\|) \leq 1-\sqrt{\left(1-k\left|e_{y}\left(\tau_{c}\right)\right|\right)^{2}+k^{2} e_{z}^{2}\left(\tau_{c}\right)} .
$$

If $\left|k e_{y}\left(\tau_{c}\right)\right| \leq 1$, then maximizing $\left|e_{y}\left(\tau_{c}\right)\right|$ will also maximize the right-hand side of (15) for a given $\left|e_{z}\left(\tau_{c}\right)\right|$. As in case 1, examination of (10) and use of the coefficient formulas in the Appendix allows us to bound $\left|k e_{y}\left(\tau_{c}\right)\right|$ via the triangle inequality $\left(\left|e_{y}\left(\tau_{c}\right)\right| \leq\left|a_{y}\right|+\left|b_{y}\right|+\left|d_{y}\right|\right)$, obtaining

$$
k\left|e_{y}\left(\tau_{c}\right)\right| \leq \frac{k e_{z}(0)}{\bar{q}}+\frac{2}{\bar{q}^{2}} .
$$

Noting again that the right-hand side is a function of $e_{z}(0)$, we consider that $c_{z} 2 \pi / q+d_{z}-\left|a_{z}\right|-\left|b_{z}\right| \leq e_{z}\left(\tau_{c}\right)$ and get

$$
k e_{z}(0) \leq \frac{\bar{q}^{3} k e_{z}\left(\tau_{c}\right)+2 \pi \bar{\lambda}^{2}+1}{\bar{q}\left(\bar{\lambda}^{2}-1\right)}
$$

where again we have the constraint that $\lambda_{2} / \lambda_{1}<k$. Substitution of (17) into (16), and (16) into (15), gives the error bound as a function of $e_{z}\left(\tau_{c}\right)$ in the case that $k\left|e_{y}\left(\tau_{c}\right)\right| \leq 1$. Maximizing the right-hand side with respect to $e_{z}\left(\tau_{c}\right)$ by minimizing the quadratic under the radical then gives

$$
\min (k\|\boldsymbol{e}(t)\|) \leq \frac{2 \pi \bar{\lambda}^{2}+1}{\bar{q}^{2}\left(\bar{\lambda}^{2}-1\right)}+\frac{2}{\bar{q}^{2}}=\beta_{2}
$$

for case 2 with $k\left|e_{y}\left(\tau_{c}\right)\right| \leq 1$. This holds for all $\bar{\lambda}$ larger than some fixed value because a monotonically decreasing (with respect to $\bar{\lambda}$ ) bound on $k\left|e_{y}\left(\tau_{c}\right)\right|$ can be found by substituting (17) into (16) and noting that $e_{z}\left(\tau_{c}\right)<1 / k$ in case 2 . If $\bar{\lambda}$ is not large enough to guarantee that this bound is less than 1 , then we appeal to the fact that $k\|\boldsymbol{e}(t)\| \leq 1$ for any termination in the circular phase as stated above.

Comparison then reveals that $\beta_{1}>\beta_{2}$ for $k\left|e_{y}\left(\tau_{c}\right)\right| \leq 1$, and $\beta_{1}>1$ for $k\left|e_{y}\left(\tau_{c}\right)\right|>1$; therefore, a conservative upper bound for all cases is $\beta=\beta_{1}$. As noted above, $\beta$ approaches zero as $\lambda_{2} / \lambda_{1}$ approaches infinity. This confirms the geometric argument that the control law drives the needle to the exact target location in the limit as $\lambda_{2} / \lambda_{1}$ approaches infinity.

\section{Simulation StUdy to VALIDATE ERROR BOUND}

We performed a set of 1000 simulations to check our theoretical upper bound against the actual minimum error for various desired target points, curvature values, and control parameters. In each simulation, a random curvature value in the range $\left[\begin{array}{ll}1 & 20\end{array}\right] \mathrm{m}^{-1}$ was assigned, and a random target point was selected such that: 1) the torus condition was satisfied, and 2) the initial target error was of the form $e=\left[\begin{array}{lll}0 & e_{y} & e_{z}\end{array}\right]^{T}$ with $e_{y} \in\left[\begin{array}{ll}-2 / k & 0\end{array}\right]$ and $e_{y} \in\left[\begin{array}{ll}0 & 2 / k\end{array}\right]$ (simulating the initial rotation to $\sigma=0$ ). Control parameters were chosen as $\lambda_{1}=1 \mathrm{~cm} / \mathrm{s}$ and $\lambda_{2}$ was determined by randomly selecting the dimensionless parameter $\bar{\lambda}=\frac{\lambda_{2}}{k \lambda_{1}}$ in the range $\left[\begin{array}{ll}1 & 100\end{array}\right]$. The kinematic model was then simulated using the control law and was ended as soon as $e_{z}(t)=0$. The magnitude of the error at the end of the simulation was recorded for each run.

The results of this study are plotted in Fig. 2, which compares the upper bound on the minimum dimensionless error given by (14) to the actual minimum errors generated by the simulations, as a function of the dimensionless group $\bar{\lambda}$. The reason for the gap between the simulation data and the upper bound plot is that the triangle inequality was used in obtaining the closed-form algebraic expression for the bound. Thus, $\beta$ is a conservative upper bound on the error. Obtaining the least upper bound would involve solving several nonlinear equations, and the answer may not be expressible in a convenient algebraic form.

\section{USE OF THE ERROR BOUND}

One reason for finding the above error bound for this control law was to verify that there are no theoretical stability or 


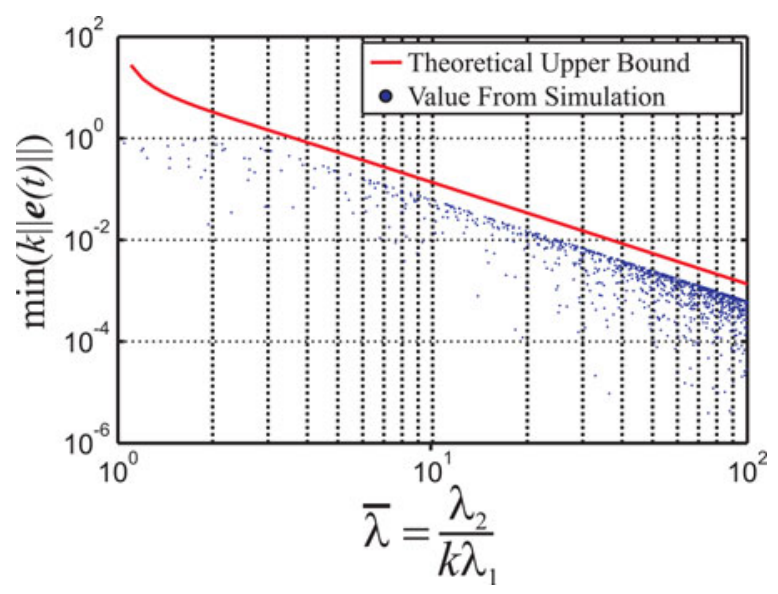

Fig. 2. Comparison of our upper bound on the minimum dimensionless error given by (14) to actual minimum errors generated by simulations of 1000 needle insertions with randomized target locations, model curvatures, and control parameters. The data and theoretical bound are plotted with respect to the dimensionless group $\bar{\lambda}$. We note that the derivation of the error bound assumes that the model is perfectly correct, but it may still be used in practice to select control parameters that are at least capable of achieving a desired accuracy. The actual accuracy in implementation will of course be affected by the structural correctness of the model and sensory limitations.

convergence issues, and that the error bound indeed approaches zero as $\lambda_{2} / \lambda_{1}$ increases to infinity.

Since the upper bound is formulated in terms of the dimensionless quantity $\bar{\lambda}$ alone, (14) and Fig. 2 are valid for a needle with any curvature and may be used as design tool for selecting appropriate control parameters to achieve a desired level of accuracy. It is important to note that the bound only provides a maximum theoretical error assuming that the model is perfectly correct. Thus, the bound should only ever be used to check that a set of control parameters at least achieves a desired accuracy in theory. For instance, if a needle's curvature is $10 \mathrm{~m}^{-1}$, then an ideal system with $\bar{\lambda} \geq 40$ will always achieve submillimeter accuracy.

The actual accuracy in implementation will of course be affected by many factors, such as error in the model parameter $k$, error in the structure of the model itself (which could arise from torsion, tissue deformation, etc.), and the accuracy of sensors and actuators. All of these will contribute to produce a larger error in any physical needle steering system.

However, the control law itself is likely to perform well to the extent that the basic structure of the model is correct (i.e., as long as the tip moves in the direction the needle shaft is pointing, and as long as the tip tangent rotates toward the asymmetric-tip direction as it is inserted). These requirements are obviously influenced by the environmental factors listed above, and our bench-top experiments in Section IX provide validation of the performance in a real system in both phantom and ex vivo biological tissue.

\section{VARIATIONS ON THE BASIC LAW}

\section{A. Linear Control in a Boundary Layer}

Many sliding mode control strategies use a linear control law near the sliding manifold in order to smooth out the dis- continuity of the switched control law and eliminate chattering behavior [24]. This is an appropriate modification to make in the case of our proposed control approach since we would like to command continuous actuator speeds. Employing a boundary layer also makes simulation of the needle system easier from a computational standpoint by making the model differential equations continuous so that they become amenable to standard routines for numerical integration of initial-value problems. We can employ the standard boundary layer approach as follows:

$$
\begin{array}{ll}
u_{1}=\lambda_{1} & \\
u_{2}=\lambda_{2} \operatorname{sign}(\sigma), & \text { if } \epsilon<|\sigma| \\
u_{2}=\lambda_{2} \sigma / \epsilon, & \text { if }|\sigma| \leq \epsilon
\end{array}
$$

where $\epsilon$ is the thickness of the boundary layer.

We note that if sensor noise is considered, temporary chattering behavior near $\sigma= \pm \pi$ is possible if $e_{x}$ happens to be sensed alternately positive and negative, which would cause the commanded rotation speed to alternate back and forth. However, we note that the system will tend to quickly break free from any such cycle because $\sigma= \pm \pi$ is never a stable system state. This issue can be further mitigated by dynamically smoothing the sensor data and completely eliminated if the commanded rotational velocity is large enough to move the system more than one noise bandwidth away from the boundary between sensor measurements.

\section{B. Limiting Total Rotation}

If we integrate the control input $u_{2}$ over time, we get the total angular displacement that has been applied to the base of the needle

$$
\theta(t)=\int_{0}^{t} u_{2}(\tau) d \tau
$$

The control laws given by (19) or (5) allow $\theta(t)$ to grow unbounded with time, but this may be undesirable if there is additional instrumentation which attaches to the base of the needle or travels through it (e.g., sensors or ablation wires).

We can modify our control policy to respect a hard limit on $\theta$ as follows:

$$
\begin{aligned}
& u_{1}=\lambda_{1} \\
& W \leftarrow 1, \quad \text { if }|\theta|>\theta_{\max } \\
& W \leftarrow 0, \quad \text { if }|\theta|<\alpha \\
& u_{2}=-\lambda_{2} \operatorname{sign}(\theta), \quad \text { if } W=1 \\
& u_{2}=\lambda_{2} \operatorname{sign}(\sigma), \quad \text { if } \epsilon<|\sigma| \text { and } W=0 \\
& u_{2}=\lambda_{2} \sigma / \epsilon, \quad \text { if }|\sigma| \leq \epsilon \text { and } W=0
\end{aligned}
$$

where $W$ is a switched variable that is initialized as $W=0$ at $t=0$ and assigned a value of 1 when the desired absolute limit $\theta_{\max }$ is violated. $W$ remains at 1 until $|\theta|$ falls below a lower threshold $\alpha$, at which point it is reassigned a value of zero. We verified this approach in simulation with $\theta_{\max }=270^{\circ}$ and $\alpha=45^{\circ}$, but we cannot guarantee that the error bound (14) will 


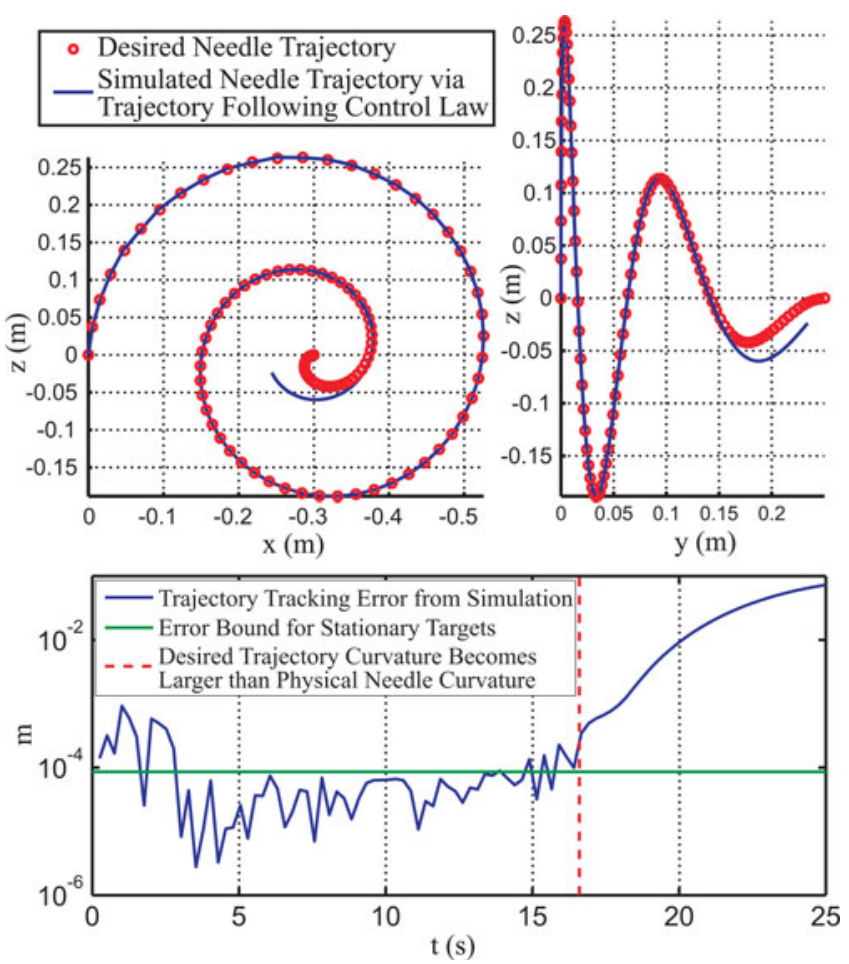

Fig. 3. Simulation of our proposed control law used to track a desired trajectory in space. The desired path spirals with a linearly decreasing radius. The simulated needle path diverges when the curvature of the desired trajectory exceeds the needle curvature ( $k=10 \mathrm{~m}^{-1}$ for this simulation).

still apply for this control law. However, the accuracy can still always be increased by increasing $\lambda_{2} / \lambda_{1}$.

\section{Tracking a Desired Trajectory}

The proposed control approach may also be used to drive the needle along a specified continuous trajectory in $\mathbb{R}^{3}$, such as might be produced as the output of a path-planning algorithm. If we define the desired path as a function of time $\boldsymbol{p}_{d}(t)$, the control law can be modified as follows:

$$
\begin{aligned}
& u_{1}=\left\|\dot{\boldsymbol{p}}_{d}\right\|+k_{p} e_{z} \\
& u_{2}=\frac{\lambda_{2}}{\lambda_{1}} \operatorname{sign}(\sigma)\left\|u_{1}\right\|
\end{aligned}
$$

where $k_{p}$ is a proportional gain coefficient.

While a formal analysis of trajectory tracking accuracy is beyond the scope of this paper, we show a test of this control law for a desired trajectory in Fig. 3. The control parameters used were $\lambda_{1}=5 \mathrm{~mm} / \mathrm{s}, \lambda_{2}=2 \pi \mathrm{rad} / \mathrm{s}$, and $k_{p}=0.5 \mathrm{~s}^{-1}$, with a boundary layer thickness of $\epsilon=1^{\circ}$. The simulated needle curvature was $k=10 \mathrm{~m}^{-1}$, and the following desired path was tracked from $t=0 \mathrm{~s}$ to $t=25 \mathrm{~s}$ :

$$
\boldsymbol{p}_{d}=\left[\begin{array}{c}
(A-\alpha t) \cos (w t)-A \\
B t^{2} \\
(A-\alpha t) \sin (w t)
\end{array}\right]
$$

where $A=300 \mathrm{~mm}, B=0.4 \mathrm{~mm} / \mathrm{s}^{2}, \alpha=12 \mathrm{~mm} / \mathrm{s}$, and $w=$ $0.5 \mathrm{rad} / \mathrm{s}$.
We note two aspects of the resulting plots in Fig. 3. First, the desired trajectory curvature increases beyond the modeled curvature of the simulated needle, illustrating the physical limitations of the system to follow a completely arbitrary curve. Second, the desired tip trajectory begins coincident with the needle tip, but misaligned with the needle tangent axis by $4.6^{\circ}$ (the needle tip frame is aligned with the global axes). As shown in Fig. 3, the trajectory tracking error during the simulation sometimes exceeds the upper bound we derived for the stationary target case. However, the plot suggests that 1) the stationary bound may represent a reasonable expectation of theoretical accuracy for the trajectory tracking case and that 2) the control approach is capable of correcting initial misalignments between the desired path and needle tangent. As in the stationary case, we hasten to add that any physical needle steering system will exhibit less accuracy because of modeling and sensing uncertainty.

\section{Considerations for Path Planning}

While the only theoretical restriction on a planned path is that it does not exceed the maximum achievable needle curvature at any point, the simulation results suggest that paths with curvatures close to the maximum may also be difficult for the control law to follow with predictable accuracy. In Fig. 3, the error begins to grow when the desired path curvature is approximately $75 \%$ of the theoretical maximum. We suggest that this could be a good guideline for path-planning considerations, acting as a heuristic to account for sensor uncertainty and physical perturbations from tissue inhomogeneity that could render a desired maximum possible curvature path unfollowable. Furthermore, there is some uncertainty in the estimation of the maximum achievable curvature for a particular application, since it will depend on tissue properties, making it desirable to plan paths of somewhat less than maximum needle curvature.

\section{EXPERIMENTAL VALIDATION}

We now describe several experiments designed to validate the effectiveness of our control law for steerable needles. We performed a number of target-hitting and trajectory following tests in both phantom tissue and ex vivo bovine liver. As noted above, the control law was completely ignorant of the needle path curvature $k$, and the accuracy of the approach is made evident by the low errors reported below.

\section{A. Benchtop Setup}

Our experimental testbed, which is shown in Fig. 4, consists of a needle insertion robot, a bevel-tipped needle, and an electromagnetic tracking system. The robot controls both needle insertion and axial rotation via DC motors, with low-level PID control implemented using a Galil DMC 4080 Motion Control unit, and high-level control implemented on a PC, which is connected to the DMC 4080 via Ethernet. The needle was a $0.86 \mathrm{~mm}$ diameter Nitinol tube with an approximately $35^{\circ}$ beveled brass tip, which was inserted 1-2 mm into the tip of the tube, and fixed in place with adhesive. We embedded a five-degree-of-freedom 


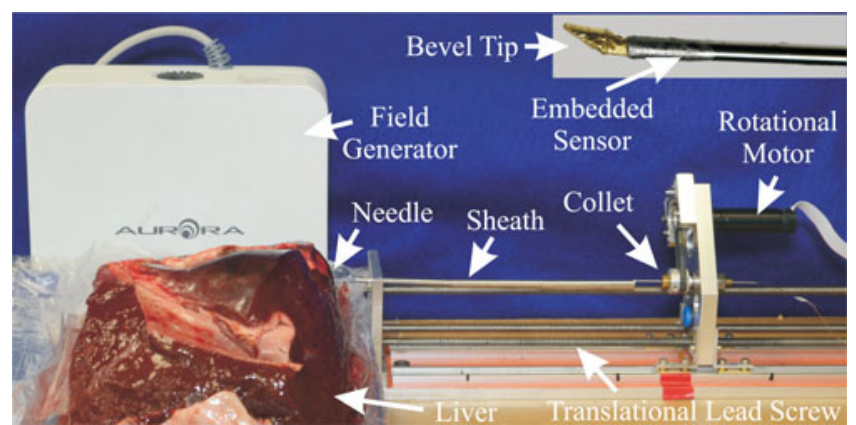

Fig. 4. Our experimental testbed consists of an electromagnetically tracked bevel-tipped needle, a 2-DOF robotic actuation unit for rotation and insertion driven by a low-level motion controller, and phantom or ex vivo tissue samples.

(DOF) magnetic tracking coil within the tip of the tube just behind the beveled tip insert. The position and tangent vector along the sensor coil was measured by the Aurora magnetic tracking system (Northern Digital, Inc., Waterloo, ON, Canada). We note that the position and tangent vector at the tip of the needle could also be acquired within other sensory frameworks, e.g., biplane flouroscopy.

As shown in Fig. 4, the robotic actuation unit contains a single carriage actuated by lead screw. The needle is gripped by a collet, which is housed in a rotary bearing attached to the carriage. The collet grips the tube and is fixed in a toothed pulley via two set screws. The pulley is actuated via belt drive by a motor attached to the carriage. Needle buckling during insertion was prevented by the fixed entry point at the front of the actuation unit and also by using an external telescoping sheath between the collet and the entry point [23].

In our tests, we used the ballistic test media SIM-TEST from Corbin Manufacturing \& Supply, Inc. as a phantom designed to simulate muscle tissue, and we also performed tests in freshly excised ex vivo bovine liver that we obtained from a local butcher shop. In order to obtain the full pose of the needle tip, we implemented a Kalman filter on the 5-DOF magnetic sensor data and the control inputs. Our filter implementation used the unicycle motion model (1) and an estimate of the needle path curvature (although not used in the control law itself), which we obtained experimentally by gathering magnetic tracker data points during an insertion without axial rotation. We least-squares fit a plane to these points and then fit a circle to the planar projections of the points, thus finding a radius of curvature of $12.8 \mathrm{~cm}$ for the phantom tissue and $40 \mathrm{~cm}$ for the ex vivo liver. A more robust estimate could be made by fitting the data from multiple insertion tests.

\section{B. Target-Hitting Phantom Experiments}

We chose target points in space expressed in coordinates of the initial tip frame and inserted the bevel-tipped needle under closed-loop control to each target location. We implemented (19) as the control law with $\lambda_{1}=5 \mathrm{~mm} / \mathrm{s}, \lambda_{2}=2 \pi \mathrm{rad} / \mathrm{s}$, and a boundary layer thickness of $\epsilon=10^{\circ}$. We selected these control parameters a priori, considering them modest and safe veloci-

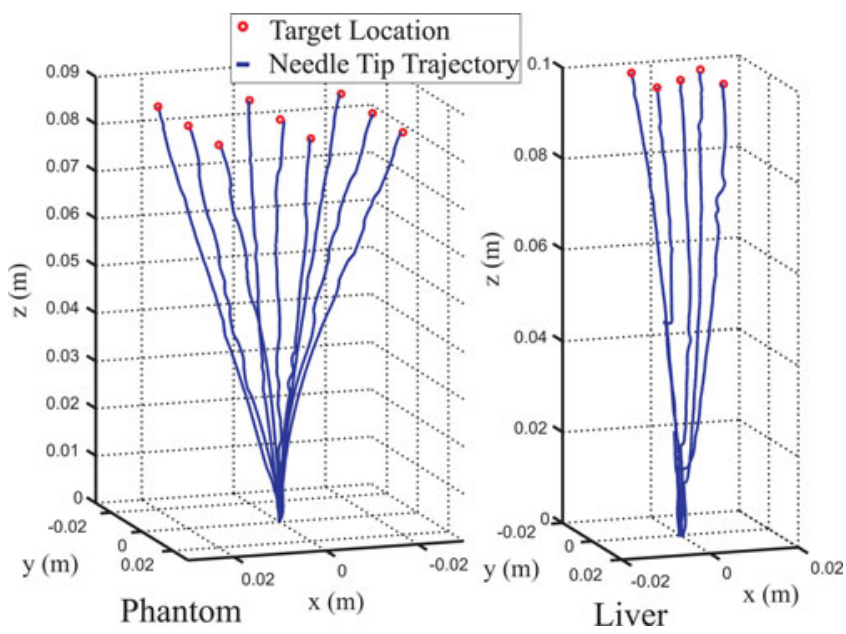

Fig. 5. Plots of the needle tip trajectory sensed by the magnetic tracker during execution of our control law in phantom tissue and ex vivo liver. The target locations are shown by the red circles.

TABLE I

EXPERIMENTAL TIP ERRORS IN PHANTOM

\begin{tabular}{|c|c|c|}
\hline Trial \# & Target Point $(\mathrm{mm})$ & Tip Error $(\mathrm{mm})$ \\
\hline 1 & {$\left[\begin{array}{lll}-20 & -20 & 85\end{array}\right]$} & 0.4 \\
\hline 2 & {$\left[\begin{array}{lll}0 & -20 & 85\end{array}\right]$} & 0.2 \\
\hline 3 & {$\left[\begin{array}{lll}20 & -20 & 85\end{array}\right]$} & 0.4 \\
\hline 4 & {$\left[\begin{array}{lll}-20 & 0 & 85\end{array}\right]$} & 0.5 \\
\hline 5 & {$\left[\begin{array}{lll}0 & 0 & 85\end{array}\right]$} & 1.3 \\
\hline 6 & {$\left[\begin{array}{lll}20 & 0 & 85\end{array}\right]$} & 0.3 \\
\hline 7 & {$\left[\begin{array}{lll}-20 & 20 & 85\end{array}\right]$} & 0.4 \\
\hline 8 & {$\left[\begin{array}{lll}0 & 20 & 85\end{array}\right]$} & 0.3 \\
\hline 9 & {$\left[\begin{array}{lll}20 & 20 & 85\end{array}\right]$} & 0.3 \\
\hline
\end{tabular}

TABLE II

EXPERIMENTAL TIP ERRORS IN LIVER

\begin{tabular}{|c|c|c|}
\hline Trial \# & Target Point $(\mathrm{mm})$ & Tip Error $(\mathrm{mm})$ \\
\hline 1 & {$\left[\begin{array}{lll}0 & 0 & 100\end{array}\right]$} & 0.4 \\
\hline 2 & {$\left[\begin{array}{lll}7.5 & 7.5 & 100\end{array}\right]$} & 0.5 \\
\hline 3 & {$\left[\begin{array}{lll}-7.5 & -7.5 & 100\end{array}\right]$} & 0.1 \\
\hline 4 & {$\left[\begin{array}{lll}-7.5 & 7.5 & 100\end{array}\right]$} & 0.8 \\
\hline 5 & {$\left[\begin{array}{lll}7.5 & -7.5 & 100\end{array}\right]$} & 0.2 \\
\hline
\end{tabular}

ties, and we used our error bound to verify that the system would be capable of achieving submillimetric error.

Each insertion was terminated when the sign of $e_{z}$ reached zero (when the error stopped decreasing). After each experiment, the needle was removed from the phantom and repositioned at a different entry point to minimize the chance of a previous needle path channel influencing the current experiment. This process was repeated for nine reachable target locations in phantom tissue, and for five target locations in liver.

The resulting trajectories are plotted in Fig. 5 (in the coordinate system of the initial tip frame). The target points and resulting tip errors (between desired point and the filtered position) at the end of each experiment are recorded in Tables I and II. The mean error was $0.5 \mathrm{~mm}$ for the nine phantom trials and $0.4 \mathrm{~mm}$ for the five liver trials. Phantom trial 5 displayed significantly higher error than the others. We executed it a second time and obtained an error of $0.2 \mathrm{~mm}$. A possible cause for the higher error is diversion of the needle trajectory by a channel in the phantom made by one of the previous insertions. 


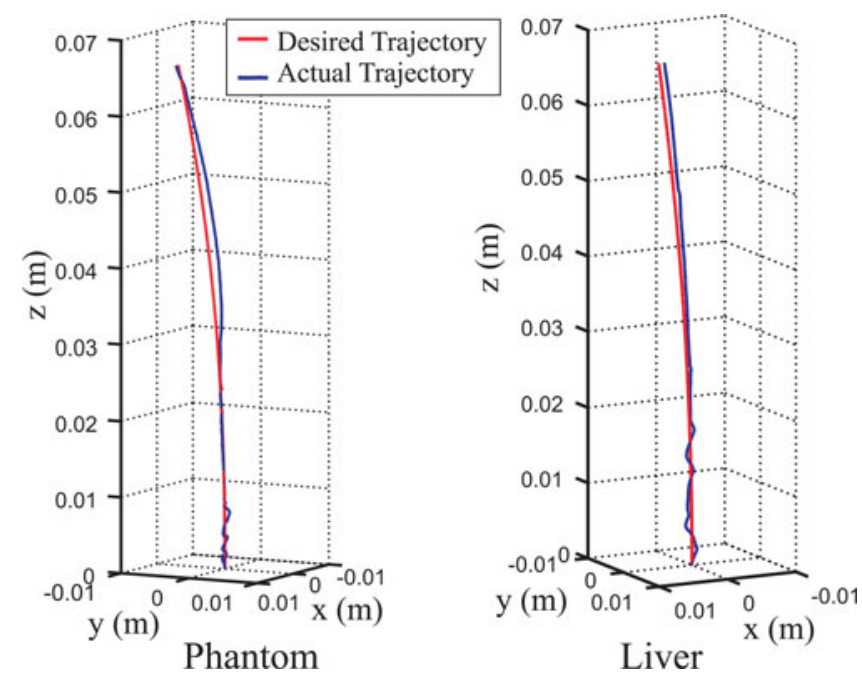

Fig. 6. Desired needle tip trajectory and the trajectory sensed by the magnetic tracker and Kalman filtered during execution of our control law in phantom and liver tissue.

Several of the liver trials had to be terminated early and retried at a different insertion point because the needle encountered an obstacle that it could not penetrate (e.g., a membrane or vessel) and buckled. This was expected since our insertion locations were chosen without knowledge of interior structures, and it illustrates the importance of path planning for needle steering problems. In some of the recorded liver trials, the needle encountered obstacles and partially buckled but was successful in penetrating them and proceeding to the target. One such disturbance in the needle trajectory can be seen in Fig. 5 in the second liver path from the left.

Overall, the submillimeter targeting error results from these experiments serve to validate the proposed control approach, and the prospect of using such an approach in clinical procedures is promising. We note that even $2-3 \mathrm{~mm}$ errors are tolerated by most surgical needle placement procedures, and human manual placement error is approximately $3-5 \mathrm{~mm}$ [25].

\section{Trajectory-Following Experiments}

We also performed trajectory-following experiments in both phantom and liver to test the control law given by (22). We used the same ratio of $\lambda_{2} / \lambda_{1}$ as in the target-hitting experiments, and we manually tuned the proportional gain to balance tracking accuracy and smoothness of the commanded insertion profile, arriving at $k_{p}=2 \mathrm{~s}^{-1}$. We chose a desired (and achievable) trajectory given by

$$
\boldsymbol{p}_{d}=\left[\begin{array}{c}
A t \sin (w t) \\
A t(\cos (w t)-1) \\
B t
\end{array}\right]
$$

where $A=0.3 \mathrm{~mm} / \mathrm{s}^{2}, B=3 \mathrm{~mm} / \mathrm{s}, w=0.05 \mathrm{rad} / \mathrm{s}$ for the phantom case, and $w=0.03 \mathrm{rad} / \mathrm{s}$ for the liver case. The control law was employed to follow this trajectory and was manually stopped after $22 \mathrm{~s}$ in each case. The resulting sensed trajectories are shown in blue in Fig. 6 with the desired trajectory superimposed in red. The resulting tip error magnitude is plotted as
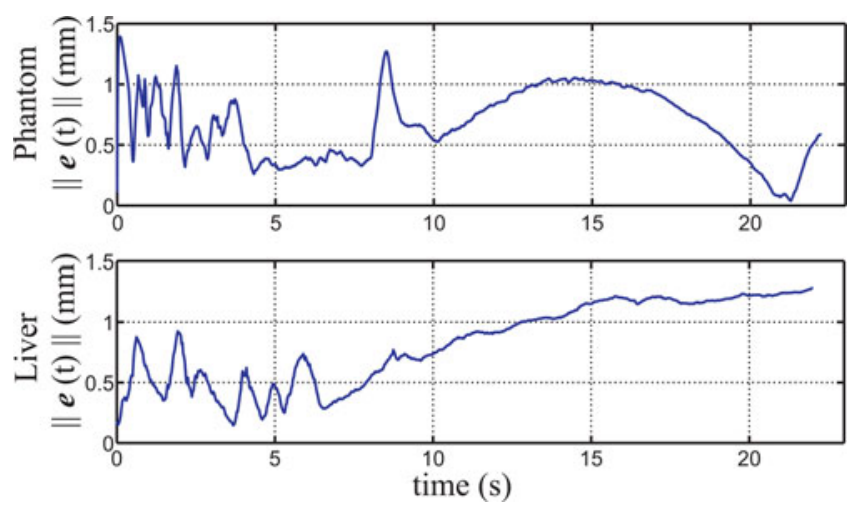

Fig. 7. Sensed error over time between the desired trajectory and actual trajectory of the needle in phantom and liver tissue.

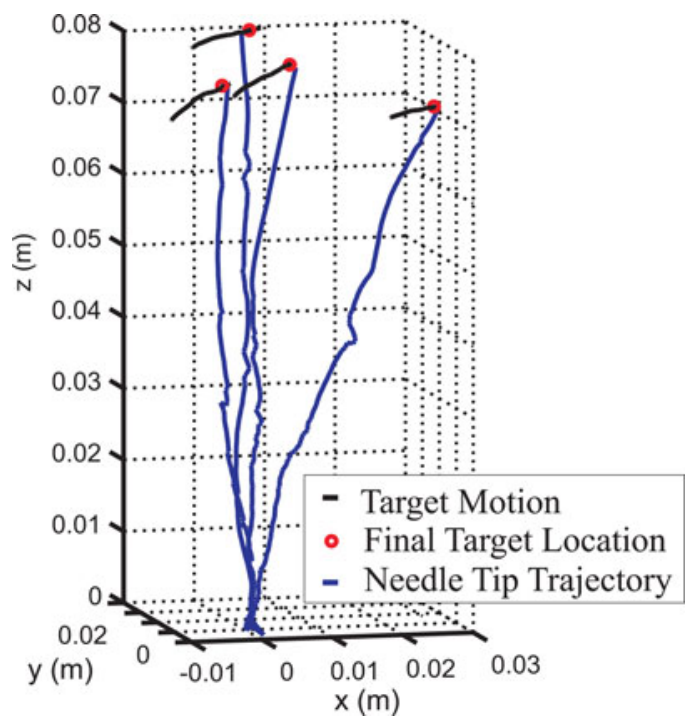

Fig. 8. Plots of the sensed needle tip trajectory during manual deformation of the phantom tissue, which caused the embedded target to move. The final target locations are shown by the red circles and the motion of the targets during insertion is shown by the black lines.

a function of insertion time in Fig. 7. The plots indicate that if trajectory error is incurred due to some disturbance, it can take some time to overcome it, even if the desired path curvature stays beneath the maximum achievable curvature. In these experiments, the maximum curvatures of the desired trajectories were $46 \%$ and $95 \%$ of their respective estimated maximum needle curvatures for the phantom and liver cases, respectively.

\section{Phantom Experiments With Deformation and Target Motion}

In the experiments described above, the targets and desired trajectories were fixed in space rather than fixed to the tissue itself. A small amount of deformation was observed during these experiments, which disturbed the needle trajectory, but the control law was still able to guide the needle to the target location. To test the ability to hit points in the tissue while it is undergoing significant deformation, we performed a second set of target-hitting experiments with a magnetically tracked coil (the "target coil") embedded within the phantom. 
TABLE III

TIP ERRORS WITH DEFORMATION

\begin{tabular}{|c|c|c|}
\hline Trial \# & Target Displacement $(\mathrm{mm})$ & Tip Error $(\mathrm{mm})$ \\
\hline 1 & 9.1 & 1.4 \\
\hline 2 & 6.5 & 0.7 \\
\hline 3 & 11.3 & 1.3 \\
\hline 4 & 10.3 & 0.9 \\
\hline
\end{tabular}

The sensed pose of the embedded 5-DOF target coil was used to define the target position $13 \mathrm{~mm}$ away from the coil along its tangent vector. The position of the coil itself was not used directly in this experiment because it is not desirable to have the needle actually hit the tracking coil. If it did, it might damage the coil, and it is also possible that two tracking coils (the one in the needle tip and the one used as the target) might interfere with one another by distorting the magnetic field if placed in very close proximity. In addition, the tissue at the target coil and at the target point $13 \mathrm{~mm}$ away from it was made to move with approximately the same displacement.

We then executed four target-hitting experimental runs where the phantom tissue was manually deformed during insertion by pushing downward on the surface near the target coil. The resulting needle tip trajectories and target motion over time are plotted in Fig. 8. The tip errors and magnitude of the target displacement during each experiment are reported in Table III.

\section{CONCLUSION}

We have presented a new category of control laws for asymmetric-tipped needles based on a sliding mode approach. We showed convergence of the basic control law within a theoretical error tolerance and experimentally validated the control approach in phantom tissue and ex vivo liver tissue, hitting target points and following desired trajectories with submillimetric average accuracy. The control law itself is independent of any model parameters (e.g., the intrinsic curvature of the needle path, tissue properties, or boundary conditions), and it performed well even in the case of significant deformation and tissue inhomogeneities in the liver. Based on the high accuracies obtained in our experimental results, in future work, we intend to replace the magnetic tracking data with information sensed from medical images and deploy our needle steering control technique in vivo.

\section{APPENDIX}

For reference, we explicitly write out the coefficients in (10) as follows:

$$
\begin{aligned}
a_{x} & =e_{y}(0) u_{2} / q+k u_{1}^{2} u_{2} / q^{3} \\
b_{x} & =e_{x}(0) u_{2}^{2} / q^{2}-e_{z}(0) k u_{1} u_{2} / q^{2} \\
c_{x} & =-k u_{1}^{2} u_{2} / q^{2} \\
d_{x} & =e_{z}(0) k u_{1} u_{2} / q^{2}-e_{x}(0) u_{2}^{2} / q^{2}+e_{x}(0) \\
a_{y} & =-e_{x}(0) u_{2} / q+e_{z}(0) k u_{1} / q \\
b_{y} & =e_{y}(0)+k u_{1}^{2} / q^{2} \\
d_{y} & =-k u_{1}^{2} / q^{2}
\end{aligned}
$$

$$
\begin{aligned}
a_{z} & =-e_{y}(0) k u_{1} / q-k^{2} u_{1}^{3} / q^{3} \\
b_{z} & =-e_{x}(0) k u_{1} u_{2} / q^{2}+e_{z}(0) k^{2} u_{1}^{2} / q^{2} \\
c_{z} & =-u_{1} u_{2}^{2} / q^{2} \\
d_{z} & =e_{x}(0) k u_{1} u_{2} / q^{2}+e_{z}(0) u_{2}^{2} / q^{2} .
\end{aligned}
$$

\section{REFERENCES}

[1] R. Lencioni, L. Crocetti, M. Pina, and D. Cioni, "Percutaneous imageguided radio frequency ablation of liver tumors," Abdominal Imag., vol. 34, no. 5, pp. 547-556, 2009.

[2] R. H. Taylor and D. Stoianovici, "Medical robotics in computer-integrated surgery," IEEE Trans. Robot. Autom., vol. 19, no. 5, pp. 765-781, Oct. 2003.

[3] S. P. DiMaio and S. E. Salcudean, "Needle insertion modeling and simulation," IEEE Trans. Robot. Autom., vol. 19, no. 5, pp. 864-875, Oct. 2003.

[4] D. Glozman and M. Shoham, "Image-guided robotic flexible needle steering," IEEE Trans. Robot., vol. 23, no. 3, pp. 459-467, Jun. 2007.

[5] V. Mallapragada, N. Sarkar, and T. K. Podder, "Robot-assisted real-time tumor manipulation for breast biopsy," IEEE Trans. Robot., vol. 25, no. 2, pp. 316-324, Apr. 2009.

[6] P. E. Dupont, J. Lock, B. Itkowitz, and E. Butler, "Design and control of concentric-tube robots," IEEE Trans. Robot., vol. 26, no. 2, pp. 209-225, Apr. 2010.

[7] D. C. Rucker, B. A. Jones, and R. J. Webster, III, "A geometrically exact model for externally loaded concentric-tube continuum robots," IEEE Trans. Robot., vol. 26, no. 5, pp. 769-780, Oct. 2010.

[8] T. R. Wedlick and A. M. Okamura, "Characterization of pre-curved needles for steering in tissue," in Proc. Annu. Int. Conf. IEEE Eng. Med. Biol. Soc., Sep. 2009, pp. 1200-1203.

[9] P. J. Swaney, J. Burgner, H. B. Gilbert, and R. J. Webster, III, "A flexurebased steerable needle: High curvature with reduced tissue damage," IEEE Trans. Biomed. Eng., vol. 60, no. 4, pp. 906-909, Apr. 2013.

[10] S. Okazawa, R. Ebrahimi, J. Chuang, S. E. Salcudean, and R. Rohling, "Hand-held steerable needle device," IEEE/ASME Trans. Mechatronics, vol. 10, no. 3, pp. 285-296, Jun. 2005.

[11] S. Y. Ko, L. Frasson, and F. R. y Baena, "Closed-loop planar motion control of a steerable probe with a programmable bevel inspired by nature," IEEE Trans. Robot., vol. 27, no. 5, pp. 970-983, Oct. 2011.

[12] J. Burgner, P. J. Swaney, T. L. Bruns, M. S. Clark, D. C. Rucker, E. C. Burdette, and R. J. Webster, III, "An autoclavable steerable cannula manual deployment device: Design and accuracy analysis," ASME J. Med. Dev., vol. 6, no. 4, pp. 041007-1-041007-7, 2012.

[13] H. B. Gilbert and R. J. Webster, III, "Can concentric tube robots follow the leader?" in Proc. IEEE Int. Conf. Robot. Autom., 2013, pp. 4866-4872.

[14] S. Misra, K. Reed, B. Schafer, K. Ramesh, and A. Okamura, "Mechanics of flexible needles robotically steered through soft tissue," Int. J. Robot. Res., vol. 29, pp. 1640-1660, 2010.

[15] V. Kallem and N. J. Cowan, "Image guidance of flexible tip-steerable needles," IEEE Trans. Robot., vol. 25, no. 1, pp. 191-196, Feb. 2009.

[16] K. Reed, A. Majewicz, V. Kallem, R. Alterovitz, K. Goldberg, N. Cowan, and A. Okamura, "Robot-assisted needle steering," IEEE Robot. Autom. Mag., vol. 18, no. 4, pp. 35-46, Dec. 2011.

[17] R. Alterovitz, M. Branicky, and K. Goldberg, "Motion planning under uncertainty for image-guided medical needle steering," Int. J. Robot. Res., vol. 27, pp. 1361-1374, 2008.

[18] M. Abayazid, R. Roesthuis, R. Reilink, and S. Misra, "Integrating deflection models and image feedback for real-time flexible needle steering," IEEE Trans. Robot., vol. 29, no. 2, pp. 542-553, Apr. 2013.

[19] J. A. Engh, D. Kondziolka, and C. N. Riviere, "Percutaneous intracerebral navigation by duty-cycled spinning of flexible bevel-tipped needles," Neurosurgery, vol. 67, no. 4, pp. 1117-1123, 2010.

[20] W. Park, J. S. Kim, Y. Zhou, N. J. Cowan, A. M. Okamura, and G. S. Chirikjian, "Diffusion-based motion planning for a nonholonomic flexible needle model," in Proc. IEEE Int. Conf. Robot. Autom., Apr. 2005, pp. 4611-4616.

[21] K. Hauser, R. Alterovitz, N. Chentanez, A. Okamura, and K. Goldberg, "Feedback control for steering needles through 3D deformable tissue using helical paths," in Proc. Robotics: Sci. Syst., Seattle, WA, USA, Jun. 2009. 
[22] V. Duindam, J. Xu, R. Alterovitz, S. Sastry, and K. Goldberg, "Threedimensional motion planning algorithms for steerable needles using inverse kinematics," Int. J. Robot. Res., vol. 29, pp. 789-800, 2010.

[23] R. J. Webster, III, J. S. Kim, N. J. Cowan, G. S. Chirikjian, and A. M. Okamura, "Nonholonomic modeling of needle steering," Int. J. Robot. Res., vol. 25, no. 5/6, pp. 509-526, May/Jun. 2006.

[24] J. Slotine and W. Li, Applied Nonlinear Control. Englewood Cliffs, NJ, USA: Prentice-Hall, 1991.

[25] E. M. Boctor, M. A. Choti, E. C. Burdette, and R. J. Webster, III, “Threedimensional ultrasound-guided robotic needle placement: An experimental evaluation," Int. J. Med. Robot. Comput. Assist. Surg., vol. 4, no. 2, pp. 180-191, 2008.

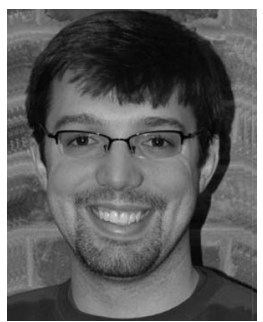

D. Caleb Rucker (S'07-M'12) received the B.S. degree in engineering mechanics and mathematics from Lipscomb University, Nashville, TN, USA, in 2006 and the Ph.D. degree in mechanical engineering from Vanderbilt University, Nashville, in 2011.

In 2013, he will join the Faculty of the University of Tennessee, Knoxville, TN, as an Assistant Professor with the Mechanical, Aerospace, and Biomedical Engineering Department, where his research will focus on robotics, mechanics, and medical applications.

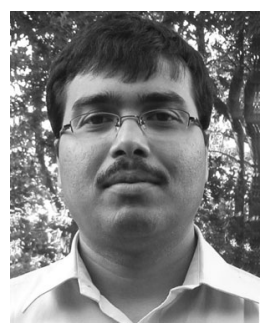

Jadav Das received the B.E. degree from Bengal Engineering and Science University, Shibpur, India, the M.Tech. degree from the Indian Institute of Technology, Kanpur, India, and the Ph.D. degree from Vanderbilt University, Nashville, TN, USA, all in mechanical engineering.

$\mathrm{He}$ is currently a Research Engineer with Rockwell Automation Inc., Shirley, NY, USA. Previously, he worked as a Technologist with the Automation Division of Tata Steel Limited, Jamshedpur, India. His research interests include the design, modeling, and control of electromechanical systems, steerable needles, robot-assisted rehabilitation, medical robotics, permanent magnets, and electromagnetic actuators.

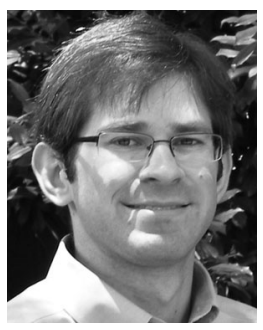

Hunter B. Gilbert (S'10) received the B.S. degree in mechanical engineering from Rice University, Houston, TX, USA, in 2010. Since then, he has been working toward the Ph.D. degree in mechanical engineering with Vanderbilt University, Nashville, TN, USA, in the Medical and Electromechanical Design Laboratory.

His research interests include medical robotics and continuum robotics.

Mr. Gilbert received the National Science Foundation Graduate Research Fellowship in 2012.

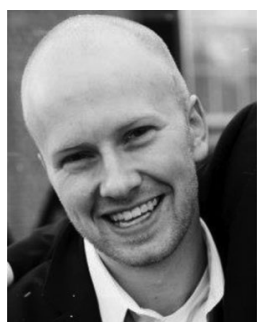

Philip J. Swaney (S'10) received the B.S. degree in mechanical engineering from The Pennsylvania State University, State College, PA, USA, in 2010. $\mathrm{He}$ is currently working toward the Ph.D. degree in mechanical engineering with Vanderbilt University, Nashville TN, USA

His current research interests include medical robotics, continuum robots, and interface design and control.

Mr. Swaney received the National Science Foundation Graduate Research Fellowship in 2012.

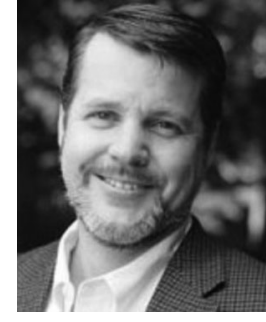

Michael I. Miga (M'98) received the B.S. and M.S degrees in mechanical engineering from the University of Rhode Island, Kingston, RI, USA, in 1992 and 1994, respectively, and the Ph.D. degree from Dartmouth College, Hanover, NH, USA, in 1998, concentrating on biomedical engineering.

He joined the Department of Biomedical Engineering, Vanderbilt University, Nashville TN, USA, in the fall of 2000, where he is currently a Professor of biomedical engineering. He is a co-founder of the Vanderbilt initiative in Surgery and Engineering Center and is the Director of the Biomedical Modeling Laboratory. His research focuses on developing and translating new interventional frameworks that use combined approaches involving computational models, instrumentation, and imaging for diagnostic and therapeutic applications.

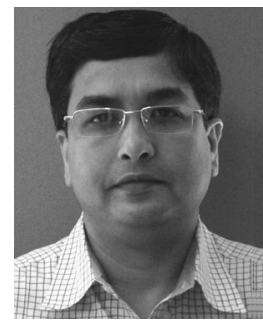

Nilanjan Sarkar (S'92-M'93-SM'04) received the $\mathrm{Ph} . \mathrm{D}$. degree in mechanical engineering and applied mechanics from the University of Pennsylvania, Philadelphia, PA, USA, in 1993

He was a Postdoctoral Fellow with Queen's University, Kingston, ON, Canada, and an Assistant Professor with the University of Hawaii, Honolulu, HI, USA, before joining Vanderbilt University, Nashville TN, USA, in 2000, where he is currently a Professor of mechanical engineering and computer engineering. His current research interests include humanrobot interaction, affective computing, dynamics, and control.

Dr. Sarkar was an Associate Editor for the IEEE TRANSACTIONS ON RовотICS

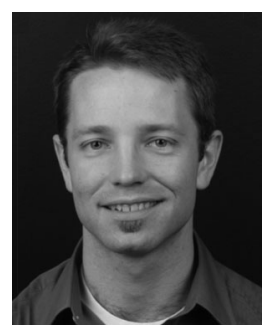

Robert J. Webster, III (S'97-M'08) received the B.S. degree in electrical engineering from Clemson University, Clemson, SC, USA, in 2002 and the M.S. and $\mathrm{Ph} . \mathrm{D}$. degrees in mechanical engineering from the Johns Hopkins University, Baltimore, MD, USA, in 2004 and 2007, respectively.

In 2008, he joined the Faculty of Vanderbilt University, Nashville, TN, USA, as an Assistant Professor of mechanical engineering, where he is a cofounder of the Vanderbilt Initiative in Surgery and Engineering and where he directs the Medical and Electromechanical Design Laboratory. His current research interests include medical robotics, image-guided surgery, and continuum robotics.

Dr. Webster received the IEEE Volz Award, for Ph.D. thesis impact, as well as the National Science Foundation CAREER Award in 2011. 